EOMmun Communication et organisation

Ory

La communication du risque

Internet, multimédia : ça change quoi, dans la réalité? de Bruno Ollivier, Paris : INRP, 2000, 154 pages, $185 \mathrm{~F}$

Élizabeth Gardère

(2) OpenEdition

Journals

Édition électronique

URL : http://journals.openedition.org/communicationorganisation/2616

DOI : 10.4000/communicationorganisation.2616

ISSN : $1775-3546$

Éditeur

Presses universitaires de Bordeaux

Édition imprimée

Date de publication : 1 novembre 2001

ISSN : 1168-5549

Référence électronique

Élizabeth Gardère, «Internet, multimédia : ça change quoi, dans la réalité ? de Bruno Ollivier, Paris : INRP, 2000, 154 pages, $185 \mathrm{~F}$ », Communication et organisation [En ligne], 20 | 2001, mis en ligne le 27 mars 2012, consulté le 19 avril 2019. URL : http://journals.openedition.org/

communicationorganisation/2616 ; DOI : 10.4000/communicationorganisation.2616

Ce document a été généré automatiquement le 19 avril 2019

(c) Presses universitaires de Bordeaux 


\title{
Internet, multimédia : ça change quoi, dans la réalité ? de Bruno Ollivier, Paris: INRP, 2000, 154 pages, $185 \mathrm{~F}$
}

\author{
Élizabeth Gardère
}

1 Évitant les réponses attendues, Bruno Ollivier pose dès le titre la question centrale de son ouvrage. Qu'est-ce que l'entrée des nouvelles technologies de l'information et de la communication peut bien modifier de plus que l'arrivée des autres machines? L'espace pédagogique est son terrain d'étude pour analyser les changements dans le mode d'accès et de structuration des connaissances suite à l'arrivée des NTIC. La mission qu'il s'est donné n'est pas de produire des réponses à l'image d'un guide d'utilisation, panacée des questions trop instrumentalisées au profit de la nouvelle économie. Son originalité et atout majeur sont d'offrir au lecteur, qu'il soit pédagogue, élève ou plus généralement au citoyen, une compréhension du phénomène Internet et multimédia par la recherche. Ce qui conduit l'auteur à remettre en cause une part de l'organisation sociale et de la communication. Son approche propose un autre mode de lecture en observant une réalité plus sociale que technologique et repère les discours qu'il soumet à une évaluation critique et une réflexion ouverte à toute observation.

2 L'auteur expose les limites et les enjeux mis en cause par la société de réseaux et prend "l'éducation et la formation comme terrain privilégié pour comprendre les enjeux et les processus mis en œuvre par l'arrivée des TIC »: l'école n'étant pas à l'abri de cette mutation. D'emblée il détermine trois postures en prenant comme discipline de référence les Sciences de l'Information et de la Communication:

3 1. Point de vue pédagogique : analyse des contenus enseignés.

4 2. Point de vue didactique : conséquences de l'usage des NTIC dans l'enseignement.

5 3. Point de vue technologique : examen des critères techniques. 
6 En formulant les bonnes questions, Bruno Ollivier fait ressortir les enjeux d'une manière d'enseigner qui est en train de se transformer dans un contexte de société de l'information, société de réseaux ou de globalisation selon les points de vue. Cette mise en perspective de la rencontre avec les NTIC dans le monde de la formation et de l'éducation met en évidence les variations des logiques d'acteurs, de leur marge d'autonomie et d'innovation.

7 Parallèlement au questionnement que suscite l'individualisation des savoirs, un certain nombre de convergences émergent: on notera le cas des procédures et dispositifs transdisciplinaires, l'entrée en formation et l'analyse des besoins ou encore les incidences institutionnelles liées à l'arrivée des TIC. L'analyse de la mutation technologique et sociale va au-delà des conséquences technologiques telles que l'interconnexion en temps réel et le démarrage de la mondialisation de l'économie. Il s'agit également de prendre en considération les regroupements d'entreprises, les rencontres des logiques d'entreprise et le croisement des cultures et métiers pour mieux comprendre l'ampleur du phénomène. 